\title{
The Theory of the Association of Free Radicals with the Hormone Melatonin in Sleep and Anaesthesia Explained
}

\author{
Dr. Muhammad Niyaz Muhammad Nuski
}

Colombo, Sri Lanka

\begin{abstract}
The sleep wake cycle has fascinated me for some time. Trying to understand how it works is an unsolved mystery. I understand that there is a lot of research in progress to solve the mystery. This is my humble attempt to explain the way this fascinating process works.In addition, I will also try to tackle the similarities of anaesthesia with sleep pertaining to its mechanism. I am a medical doctor by profession, but writing research papers is still fairly new to me. In fact, this is my very first attempt. I apologize for the lack of context in this regard.
\end{abstract}

Sleep is a naturally recurring state of mind and body, characterized by altered consciousness, relatively inhibited sensory activity, inhibition of nearly all voluntary muscles, and reduced interactions with surroundings. It is characterized by a decreased response to stimuli as compared to wakefulness. There are two types of sleep, namely REM sleep and non-REM sleep. Non-REM sleep is where the heart rate and temperature drops and while the brain uses less energy as opposed to REM sleep characterized by desynchronized and fast brain waves, eye movements, loss of muscle tone and suspension of homeostasis.The brain is known to shift in between REM and non-REM phases during the process of sleep.

Free radicals are well known to be present in the brain. They are molecules with a single electron and can cause damage to any body cells or mechanisms including of those in the brain. At the end of the day after going through a lot of stress, free radicals accumulate in the brain and body. Their presence is damaging and harmful. Also, it is well known that the levels of the hormone melatonin increases during sleep. It is known to cause sleepiness. It is in fact used to treat insomnia. It is also known to have good anti-oxidant properties. Keeping these two in mind, it is good enough to speculate the connection in between the free radicals and melatonin levels in the brain. A higher level of free radicals stimulates the secretion of melatonin thereby kicking off the process at which the free radicals are removed. I suggest that there is a threshold level at which melatonin is secreted. When the level of free radicals reach this threshold level, it stimulates the secretion of melatonin. This also explains the fact that sleep deprivation also causes loss of memory and grouchy mood. Free radicals can damage the brain cells which form the memory pathway leading to loss of memory. The damage caused by these free radicals can be felt as what I refer to as 'pain in the brain' characterized by the grouchy mood. The suprachiasmatic nucleus is known to have a direct neurological connection to the eye and it is known that this helps in the regulation of the circadian clock by secretion of melatonin. However, I think that this connection to the eye is a mere process of keeping a human being awake throughout the day or in other words it is a lesser important process by which the circadian rhythm is controlled. This is why we have some people stay up at night and sleep during the day and still function perfectly in a normal way.

The way to test my hypothesis is as follows. First, the level of both free radicals and melatonin throughout a 24 hour period should be tested and plotted, thereby the direct association of the level of melatonin with the level of free radicals can be proved and explained. Different time zones, lighting options, level of stress at work and other related parameters must be considered. Secondly, a well devised experiment must be used to test the stimulation of the suprachiasmatic nucleus by free radicals thereby proving the secretion of melatonin is also controlled by free radical stimulation.

This mechanism will also explain the shifting in between the REM and non-REM phases of sleep. Alteration in the levels of melatonin will lead to either increased activity or decreased activity in the brain. In the REM phase of sleep, the levels of free radicals increase and so the level of melatonin increases, causing the brain to drop back into the non-REM phase. It explains wakefulness as well. As the length of the sleep increases the level of free radicals decrease and thereby the level of melatonin decreases as well leading to the brain to first go into REM sleep and then finally wakefulness once the threshold has been reached.

At this point, what we begin to understand is that stress of the brain plays a major role. Increased levels of stress, increases the level of free radicals and stimulates the secretion of melatonin leading to sleep. This is why depressed people with high stress levels sleep regularly. In anaesthesia, neurotransmission is blocked leading to increased brain stress and as before leading to sleep. But in this case wakefulness is not possible as in normal sleep because stimuli to the suprachiasmatic nucleus from the eye and from other ways possible is blocked as well. So, once the effect of anaesthesia wears off, normal sleep kicks in and once the threshold has been reached wakefulness occurs while the body still recovers from the effect of anaesthesia.Anaesthesia speeds up the normal process of sleep. Exercise causes stress by increasing the level of free radicals leading to sleep. The flow chart below shows how the process works in a concise manner. 


\section{International Journal of Science and Research (IJSR) \\ ISSN (Online): 2319-7064}

Index Copernicus Value (2016): 79.57 | Impact Factor (2015): 6.391

Anaesthesia $\rightarrow$ Increased stress $\rightarrow$ Increased level of free radicals $\rightarrow$ Stimulation and secretion of melatonin $\rightarrow$ Threshold reached $\rightarrow$ Sleep $\rightarrow$ Anti oxidation $\rightarrow$ Decreased level of free radicals $\rightarrow$ Decreased level of melatonin $\rightarrow$ Threshold reached $\rightarrow$ Wakefulness.

Therefore, sleep is a process by which the body clears itself from the harmful effects of free radicals by the secretion of melatonin, wakefulness is when the level of melatonin is decreased because the level of free radicals have decreased and anaesthesia is simply speeding up of the normal sleep wake cycle of the body by increasing the level of stress. This process, can explain almost all processes leading to sleep and also helps to tackle all the unsolved mysteries of sleep if applied appropriately. I welcome, research to prove my theory correct and would love to assist in its further development.

Volume 6 Issue 12, December 2017

www.ijsr.net 